\title{
Extreme-Scale High-Order WENO Simulations of 3-D Detonation Wave with 10 Million Cores
}

YING CAI, Chinese Academy of Sciences

YULONG AO and CHAO YANG, Peking University

WENJING MA and HAITAO ZHAO, Chinese Academy of Sciences

\begin{abstract}
High-order stencil computations, frequently found in many applications, pose severe challenges to emerging many-core platforms due to the complexities of hardware architectures as well as the sophisticated computing and data movement patterns. In this article, we tackle the challenges of high-order WENO computations in extreme-scale simulations of 3D gaseous waves on Sunway TaihuLight. We design efficient parallelization algorithms and present effective optimization techniques to fully exploit various parallelisms with reduced memory footprints, enhanced data reuse, and balanced computation load. Test results show the optimized code can scale to 9.98 million cores, solving 12.74 trillion unknowns with 23.12 Pflops double-precision performance.
\end{abstract}

CCS Concepts: • Algorithms $\rightarrow$ Massively parallel computing; • Computer systems organization $\rightarrow$ Heterogeneous Many-core architectures; • Stencil $\rightarrow$ High-order;

Additional Key Words and Phrases: WENO method, Detonation waves, Sunway TaihuLight, many-core computing, computation reuse

\section{ACM Reference format:}

Ying Cai, Yulong Ao, Chao Yang, Wenjing Ma, and Haitao Zhao. 2018. Extreme-Scale High-Order WENO Simulations of 3-D Detonation Wave with 10 Million Cores. ACM Trans. Archit. Code Optim. 15, 2, Article 26 (June 2018), 21 pages.

https://doi.org/10.1145/3209208

\section{INTRODUCTION}

Gaseous wave detonations are widely found in a large variety of applications including the simulations of accelerating flames in tubes [3], industrial explosion accidents [51], coal gas explosions [47], and so on. Studying the behaviors of detonated waves with the tools of numerical simulation has been well acknowledged as an important way to resolve the complicated wave structures.

This work was supported in part by National Key R\&D Plan of China (Grant No. 2016YFB0200603) and National Natural Science Foundation of China (Grant No. 91530323).

Authors' addresses: Y. Cai, Institute of Software, Chinese Academy of Sciences, Beijing 100190, China, and University of Chinese Academy of Sciences, Beijing 100049, China; email: caiying13@iscas.ac.cn; Y. Ao and C. Yang (corresponding author), CAPT and CCSE, School of Mathematical Sciences \& NELVT, Peking University, Beijing 100871, China; emails: aoyulong@outlook.com, chao_yang@pku.edu.cn; W. Ma and H. Zhao, Institute of Software \& State Key Laboratory of Computer Science, Chinese Academy of Sciences, Beijing 100190, China; emails: \{wenjing, haitao\}@iscas.ac.cn.

Permission to make digital or hard copies of all or part of this work for personal or classroom use is granted without fee provided that copies are not made or distributed for profit or commercial advantage and that copies bear this notice and the full citation on the first page. Copyrights for components of this work owned by others than ACM must be honored. Abstracting with credit is permitted. To copy otherwise, or republish, to post on servers or to redistribute to lists, requires prior specific permission and/or a fee. Request permissions from permissions@acm.org.

(c) 2018 ACM 1544-3566/2018/06-ART26 \$15.00

https://doi.org/10.1145/3209208 
Although a considerable amount of research has been devoted to the numerical simulation of wave detonations, most were done either on 2D problems $[44,46,49,56]$ or $3 \mathrm{D}$ problems at very small scales $[6,10,46,48]$. To better understand the complicated wave motions generated in the course of detonation, high resolution simulations of real-world 3D problems have become an urgent demand. Therefore, it is of crucial importance to design highly scalable algorithms for extreme-scale simulations of 3D gaseous wave detonations on state-of-the-art computing platforms.

Unlike many fluid dynamics problems, detonation waves are usually supersonic and contain rapidly traveling shock waves with sharp fronts [36, 40, 48]. Low-order (second-order or less) numerical methods such as the Godunov [17] or the Roe schemes [34], though enjoying certain advantages such as being free of artificial oscillations, often excessively smear out some of the physically significant sharp waves, and fail to resolve sophisticated fine wave structures such as vortices and acoustic waves [38]. On the other hand, high-order methods, such as the weighted essentially non-oscillatory (WENO) schemes [19,23], are able to achieve arbitrary high-order accuracy in smooth regions while maintaining sharp, non-oscillatory discontinuity transitions. However, as compared to the traditional low-order stencils, high-order WENO stencils may cause issues on modern supercomputers because of the extra complexities in the computing and data movement patterns.

With the rapid development of high-performance computing technologies, many-core architectures have become a competitive choice to build supercomputers; examples include the Graphic Processing Units (GPUs) adopted in Titan and Piz Daint, the Intel Xeon Phis employed by Tianhe-2 and Oakforest-PACS, and, more recently, the SW26010 processors equipped in Sunway TaihuLight. In fact, half of the top 10 machines on the recent Top500 List [26] make use of many-core based processors. Optimizing stencil computations on such kind of platforms is increasingly difficult as compared to single or multi-core based systems. Despite the fact that numerous efforts have been made to improve the performance of stencil computations on many-core based architectures such as GPUs [30, 37, 39, 50], Intel Xeon Phis [42, 43, 52, 53], and SW26010 [1, 14, 31], most of the works are focused on low-order stencils. For high-order stencils such as WENO, additional difficulties may arise when implemented on modern many-core based systems, such as the exhaustive usage of local cache due to the widened stencil pattern, the redundant computation caused by the enlarged halo area, and the severe load imbalance resulted from the complicated flux computations.

Announced in June, 2016, Sunway TaihuLight is the first computer with a peak performance of over 100 Pflops. Based on the Chinese home-grown heterogeneous many-core processor, SW 26010, it can provide a massively parallel computing capability comprised of over 10 million cores [16]. Due to some of its hardware features, such as the complex heterogeneous many-core architecture, the relatively low memory bandwidth and the relatively small local cache space, it is even more challenging to make a successful deployment of applications relying on high-order stencil computations. To tackle these challenges, in this article, we conduct an in-depth study on the performance optimizations of the high-order WENO stencil computations for the extremescale simulations of 3D detonation waves on Sunway TaihuLight. In particular, we propose (1) a dependency-reduced blocking algorithm to fully exploit the on-chip parallelism with improved memory efficiency, (2) a conflict-free data exchanging mechanism together with a load-balanced collaborative computing approach to avoid redundant computations, and (3) and a componentreused vectorization strategy to increase the instruction-level parallelism. By taking advantage of the above technologies, our optimized WENO stencil computation code for gaseous wave detonation simulation can scale on Sunway TaihuLight in nearly an ideal way with up to 9.98 million cores, sustain a 23.12 Pflops performance in double precision, and solve problems with up to 12.74 trillion unknowns. 


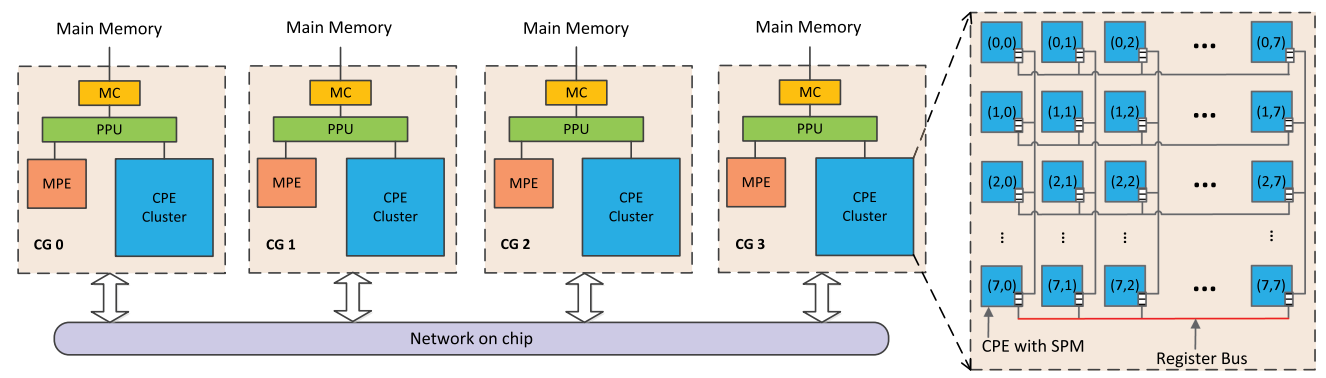

Fig. 1. The general architecture of the SW26010 processor. Each CG includes one MPE, one CPE cluster, one protocol processing unit (PPU), and one memory controller (MC). These four CGs are connected via the network on chip (NOC). The shared memory space within each CG is connected to the MPE and CPE cluster through the MC.

The remainder of this article is organized as follows. In Section 2, we give a short overview of the Sunway TaihuLight supercomputer. In Section 3 we introduce the equations for wave detonation problems and the finite difference WENO discretization. Following that, we present in Section 4 the major algorithms and some key optimization strategies for the high-order WENO computations on Sunway TaihuLight. Analysis on reductions of floating-point operations and memory footprints by utilizing the optimization strategies is carried out in Section 5 . Results for correctness validation and performance evaluation are provided in Section 6. Some related work can be found in Section 7. The article is concluded in Section 8.

\section{OVERVIEW OF SUNWAY TAIHULIGHT}

As the world's fastest supercomputer to date, Sunway TaihuLight has a theoretical peak performance of 125 Pflops and a sustained HPL Performance of 93 Pflops [16]. It consists of 40,960 compute nodes, each of which is a Chinese home-grown SW26010 processor. The general architecture of the heterogeneous many-core processor is shown in Figure 1. In each processor, there are four core groups (CGs), each of which consists of one management processing element (MPE) and 64 computing processing elements (CPEs), organized as an $8 \times 8 \mathrm{CPE}$ cluster. The MPE and CPE are both 64-bit RISC cores with 256-bit vector units but play different roles in the system. The MPE is in charge of management, task schedule, and data communication, while the CPE is designed to maximize the aggregated computing throughput.

Each CPE has its own 16KB L1 instruction cache and a 64KB scratch pad memory (SPM), which can be configured as either a user-controlled fast buffer or a software-emulated cache for automatic data caching. The user-controlled SPM of each CPE is usually called the local device memory (LDM), which can be treated as a private memory space. Each CG has its own main memory space that can be accessed by both the MPE and CPE cluster. The SW26010 processor is also equipped with direct memory access (DMA) channels to support data movement between the main memory and the LDM efficiently. By properly utilizing DMA to prepare data, the CPEs can access the data in the LDM instead of the main memory. To efficiently exchange data among different CPEs, a low-latency on-chip register data communication mechanism is provided by SW26010. Sunway TaihuLight supports mainstream parallel programming models such as MPI, OpenMP, and OpenACC. A customized lightweight accelerated thread library called Athread [16] is also provided for thread-level parallelization. We employ the hybrid program model of MPI+Athread to make full utilization of the computing capability. 


\section{EQUATIONS AND DISCRETIZATIONS}

\subsection{The Euler Equations and Numerical Discretizations}

The governing equations for the gaseous wave detonations can be represented by the compressible Euler equations with a source term for chemical reactions [48]:

$$
\begin{gathered}
\frac{\partial \mathbf{u}}{\partial t}+\frac{\partial \mathbf{f}(\mathbf{u})}{\partial x}+\frac{\partial \mathbf{g}(\mathbf{u})}{\partial y}+\frac{\partial \mathbf{r}(\mathbf{u})}{\partial z}=\mathbf{s}, \\
\mathbf{u}=(\rho, \rho u, \rho v, \rho w, \rho E, \rho Y)^{T}, \\
\mathbf{f}(\mathbf{u})=\left(\rho u, \rho u^{2}+p, \rho u v, \rho u w, \rho u(E+p / \rho), \rho u Y\right)^{T}, \\
\mathbf{g}(\mathbf{u})=\left(\rho v, \rho v u, \rho v^{2}+p, \rho v w, \rho v(E+p / \rho), \rho v Y\right)^{T}, \\
\mathbf{r}(\mathbf{u})=\left(\rho w, \rho w u, \rho v w, \rho w^{2}+p, \rho w(E+p / \rho), \rho w Y\right)^{T}, \\
\mathbf{s}(\mathbf{u})=(0,0,0,0,0, \omega)^{T},
\end{gathered}
$$

where $\rho$ is the density, $u, v, w$ are the corresponding fluid velocities of the $x, y, z$ components in the Cartesian coordinate, $E$ is the total energy for a unit mass, $p$ is the pressure, $q$ is the heat release of reaction, $\gamma>1$ is the specific heat ratio and $Y$ denotes the reactant mass fraction. The equation of state is $E=\frac{p}{(\gamma-1) \rho}+\frac{1}{2}\left(u^{2}+v^{2}+w^{2}\right)+q Y$, and the source term is assumed to be in an Arrhenius form $\omega=-\widetilde{K} \rho Y e^{-\widetilde{T} / T}$, where $T=p / \rho$ is the temperature, $\widetilde{T}$ and $\widetilde{K}$ are both constants.

Denote $\mathrm{U}$ and $\mathcal{L}$ as the solution vector and the spatial operator at the discretized level. We have a semi-discretized system in the form of

$$
\frac{d}{d t} \mathbf{U}=\mathcal{L}(\mathbf{U})
$$

which is integrated in time by using the third-order TVD Runge-Kutta scheme [18]

$$
\begin{aligned}
\mathbf{U}^{(1)} & =\mathbf{U}^{n}+\Delta t \mathcal{L}\left(\mathbf{U}^{n}\right), \\
\mathbf{U}^{(2)} & =\frac{3}{4} \mathbf{U}^{n}+\frac{1}{4} \mathbf{U}^{(1)}+\frac{1}{4} \Delta t \mathcal{L}\left(\mathbf{U}^{(1)}\right), \\
\mathbf{U}^{n+1} & =\frac{1}{3} \mathbf{U}^{n}+\frac{2}{3} \mathbf{U}^{(2)}+\frac{2}{3} \Delta t \mathcal{L}\left(\mathbf{U}^{(2)}\right),
\end{aligned}
$$

where $n$ represents the time level.

Provided a uniform mesh with values on all mesh cells indexed by subscript $(i, j, k)$, the spatially discretized results are calculated as

$$
\begin{aligned}
\mathcal{L}(\mathbf{U})_{i, j, k}= & -\frac{1}{\Delta x}\left[\hat{\mathbf{f}}(\mathbf{U})_{i+\frac{1}{2}, j, k}-\hat{\mathbf{f}}(\mathbf{U})_{i-\frac{1}{2}, j, k}\right] \\
& -\frac{1}{\Delta y}\left[\hat{\mathbf{g}}(\mathbf{U})_{i, j+\frac{1}{2}, k}-\hat{\mathbf{g}}(\mathbf{U})_{i, j-\frac{1}{2}, k}\right] \\
& -\frac{1}{\Delta z}\left[\hat{\mathbf{r}}(\mathbf{U})_{i, j, k+\frac{1}{2}}-\hat{\mathbf{r}}(\mathbf{U})_{i, j, k-\frac{1}{2}}\right]+\mathbf{s}_{i, j, k},
\end{aligned}
$$

where $\hat{\mathbf{f}}, \hat{\mathbf{g}}, \hat{\mathbf{r}}$ are numerical fluxes along the $x, y$, and $z$ directions, respectively, and $\Delta x, \Delta y, \Delta z$ are the corresponding mesh sizes.

To achieve high-order accuracy, we employ a fifth-order finite difference WENO scheme [19] for spatial discretization. At a given point representing the center of a mesh cell, the scheme adopted here leads to a 3D 19-point stencil, as shown in the left panel of Figure 2.

\subsection{WENO Stencil}

It is easy to see that the finite difference WENO scheme can be split dimension by dimension. And the single-dimension WENO stencils along the $x, y, z$ directions are similar to each other. 

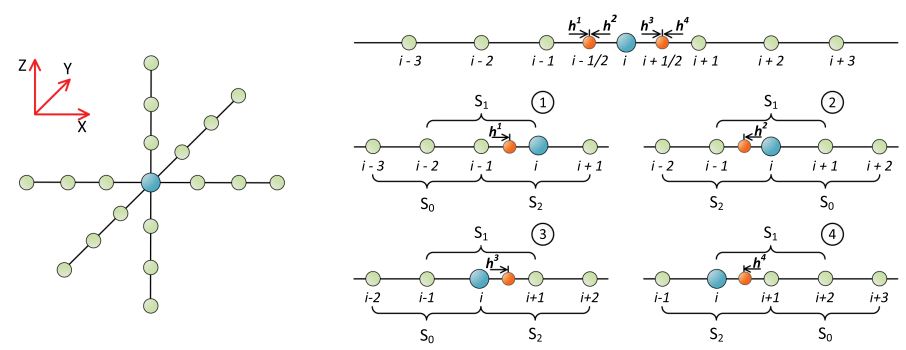

Fig. 2. Illustrations of the 3D fifth-order finite difference WENO 19-point stencil (left) and the stencil computation along $x$-direction (right). As shown in the right panel, the 7-point fifth-order finite difference WENO stencil can be decomposed into four 5 -point stencils.

Therefore, we will take the $x$-direction as an example to show how the WENO stencil,

$$
\mathbf{d}_{i}:=\frac{1}{\Delta x}\left(\hat{\mathbf{f}}(\mathbf{U})_{i+\frac{1}{2}}-\hat{\mathbf{f}}(\mathbf{U})_{i-\frac{1}{2}}\right),
$$

is computed. The subscript $j, k$ will be ignored for simplicity. The WENO algorithm along the $x$-direction for each stencil cycle is shown in Algorithm 1, where $n$ is the total number of mesh cells, $\alpha$ is the characteristic velocity, and $L$ and $R$ are, respectively, the left and right eigenvectors [19]. As shown in Algorithm 1, the WENO algorithm consists of three loops: (1) Lax-Friedrichs splitting (lines 2-5), (2) flux computation (lines 7-17), and (3) final state update (lines 19-21). In the first loop, a Lax-Friedrichs splitting is performed to get $\mathbf{f}_{i}^{ \pm}$. As for the second loop, after three sub-procedures including left/right eigen-matrix formation [38], fifth-order WENO reconstruction [19], and physical space projection, numerical fluxes $\mathbf{h}_{i \pm 1 / 2}^{ \pm}$at the mesh cell interfaces are available. Then the final state $\mathbf{d}_{i}$ can be updated based on these numerical fluxes. Among the whole procedure, the second loop is the most time-consuming part.

To be specific, the detailed computation pattern for a given mesh point $x_{i}$ is illustrated in the right panel of Figure 2 . As shown in the first row of the right panel, four numerical fluxes $\mathbf{h}_{i \pm 1 / 2}^{ \pm}$are computed to approximate the differential in the $x$-direction of node $x_{i}$. We denote the four numerical fluxes as $\mathbf{h}^{1}=\mathbf{h}_{i-1 / 2}^{+}, \mathbf{h}^{2}=\mathbf{h}_{i-1 / 2}^{-}, \mathbf{h}^{3}=\mathbf{h}_{i+1 / 2}^{+}, \mathbf{h}^{4}=\mathbf{h}_{i+1 / 2}^{-}$for simplicity. The second and third rows show how the numerical fluxes are calculated based on the fifth-order WENO reconstruction schemes [19]. In the WENO reconstruction procedure, three sub-stencils named $S_{0}, S_{1}, S_{2}$ are used to approximate $\mathbf{v}_{i \pm \frac{1}{2}}^{ \pm}$, and the final approximation is taken as a convex combination of the three approximations with nonlinear weights. Then $\mathbf{h}_{i \pm \frac{1}{2}}^{ \pm}$are obtained by multiplying the right eigenvector matrix with $\mathbf{v}_{i \pm \frac{1}{2}}^{ \pm}$. Taking $\mathbf{h}^{3}=\mathbf{h}_{i+1 / 2}^{+}$as an example, the fifth-order WENO reconstruction scheme is as follows:

$$
\begin{aligned}
& \left(\mathbf{v}_{i+\frac{1}{2}}^{+}\right)^{0}=\frac{1}{3} L \mathbf{f}_{i-2}^{+}-\frac{7}{6} L \mathbf{f}_{i-1}^{+}+\frac{11}{6} L \mathbf{f}_{i}^{+}, \\
& \left(\mathbf{v}_{i+\frac{1}{2}}^{+}\right)^{1}=-\frac{1}{6} L \mathbf{f}_{i-1}^{+}+\frac{5}{6} L \mathbf{f}_{i}^{+}+\frac{1}{3} L \mathbf{f}_{i+1}^{+}, \\
& \left(\mathbf{v}_{i+\frac{1}{2}}^{+}\right)^{2}=\frac{1}{3} L \mathbf{f}_{i}^{+}+\frac{5}{6} L \mathbf{f}_{i+1}^{+}-\frac{1}{6} L \mathbf{f}_{i+2}^{+}, \\
& \left(\mathbf{v}_{i+\frac{1}{2}}^{+}\right)=\omega_{0}\left(\mathbf{v}_{i+\frac{1}{2}}^{+}\right)^{0}+\omega_{1}\left(\mathbf{v}_{i+\frac{1}{2}}^{+}\right)^{1}+\omega_{2}\left(\mathbf{v}_{i+\frac{1}{2}}^{+}\right)^{2}, \\
& \left(\mathbf{h}_{i+\frac{1}{2}}^{+}\right)=\alpha_{i} R\left(\mathbf{v}_{i+\frac{1}{2}}^{+}\right),
\end{aligned}
$$

where the nonlinear weights $\omega_{j} \geq 0$ are variable-coefficients decided by the smoothness of the corresponding sub-stencils, more details can be found in Reference [38]. Therefore, the 7-point 


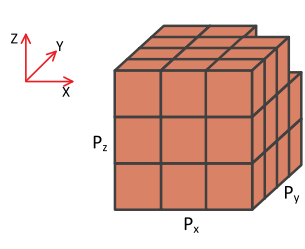

(1)Process-level partition

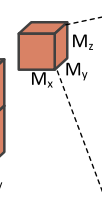

(2) CG-level blocking

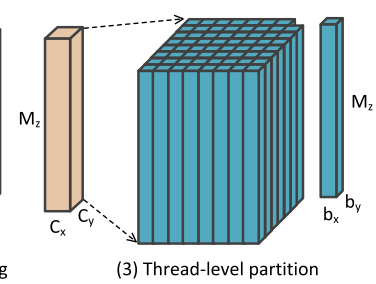

(3) Thread-level partition

Fig. 3. Illustrations of the hierarchical domain partition strategy: (1) process-level partition, (2) CG-level blocking, and (3) thread-level partition.

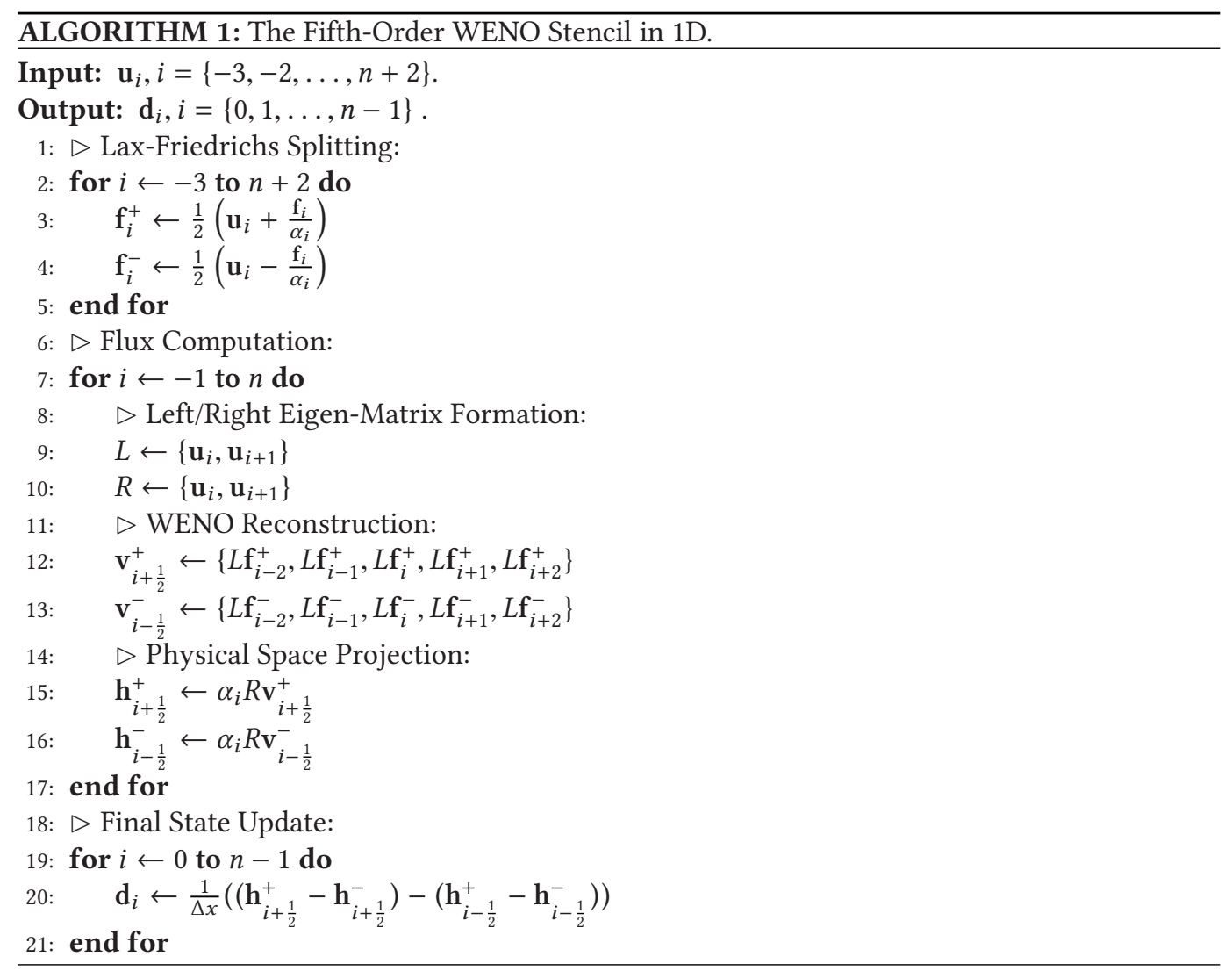

fifth-order finite difference WENO stencil along the $x$-direction can be decomposed into four 5point stencils.

\section{PARALLELIZATION AND OPTIMIZATION}

To implement the WENO stencil computations on Sunway TaihuLight, we employ a hierarchical domain partition strategy for parallelization, as illustrated in Figure 3. All unknowns are stored as 3-D arrays with the $x-y-z$ order ( $x$ is the innermost dimension). At the process level, we decompose the whole computational domain into $P_{x} \times P_{y} \times P_{z}$ partitions, with each assigned an MPI process running on a single CG. After that, each CG owns a subdomain of size $M_{x} \times M_{y} \times M_{z}$, where $M_{x}$, 


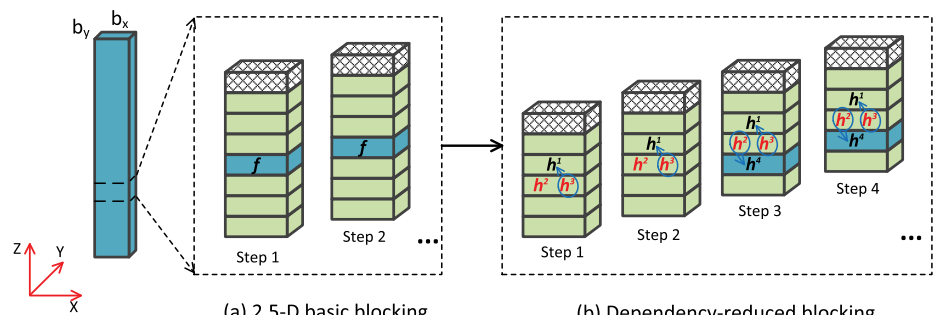

Fig. 4. Illustrations of the dependency-reduced blocking algorithm, with a comparison to the 2.5D basic blocking method.

$M_{y}, M_{z}$ correspond to the sizes in the $x, y, z$ direction, respectively. Halo information is exchanged among processes before computing the stencils in each subdomain. At the CG level, we further partition the subdomain along $x$ and $y$ axes into data chunks of size $C_{x} \times C_{y} \times M_{z}$. Each CG would iterate over these data chunks to finish the whole stencil computation. At the thread level, the data chunk is further decomposed along the $x$ and $y$ axes into blocks of size $b_{x} \times b_{y} \times M_{z}$, each of which is assigned to one of the $64 \mathrm{CPEs}$ within the CG. The block size $b_{x}, b_{y}$ will be discussed later to maximize the sustained performance. In what follows, we will mainly focus on discussing the optimization techniques inside a CG, which is the most important part of the overall performance optimization.

\subsection{Dependency-Reduced Blocking Algorithm}

A 2.5D blocking strategy is employed to fully exploit the on-chip parallelism with enhanced data locality. Although the CPE can access data directly from the main memory, the long latency of main memory access could degrade the performance severely. Therefore, we use the fast DMA channel to efficiently move the data between the main memory and the LDM. For each CPE thread, we load a suitable $z$-plane of the thread block into the LDM via DMA, and perform the computation plane by plane afterwards. Although the 2.5D basic blocking method can help exploit parallelism, the wide stencil width makes it a big burden for the limited LDM, and the computation load for each thread is still heavy.

With careful analysis, we find that there are opportunities to overlap the flux computations in adjacent planes. As shown in Figure 4, to compute the results on a given 2D plane, the 2.5D basic blocking strategy requires us to load seven adjacent planes into the LDM according to the 19-point WENO stencil, during which, four fluxes for the computed plane in $z$-direction are computed. The computation of the four intermediate fluxes can be split into three steps: (1) compute $\mathbf{h}^{1}$, (2) compute $\mathbf{h}^{2}, \mathbf{h}^{3}$, and (3) compute $\mathbf{h}^{4}$. In each step, only 5 points instead of 7 are needed. It implies that we can compute a series of intermediate numerical fluxes based on the five-layer reconstruction instead of updating the final state directly from the seven-layer stencil. Moreover, let $\mathbf{h}_{k}^{1}, \mathbf{h}_{k}^{2}, \mathbf{h}_{k}^{3}$, and $\mathbf{h}_{k}^{4}$ denote the four intermediate fluxes of the $k$-th plane; it can be observed that $\mathbf{h}_{k+1}^{1}=\mathbf{h}_{k}^{3}, \mathbf{h}_{k+1}^{2}=\mathbf{h}_{k}^{4}$.

Based on the above observations, we propose an improved dependency-reduced blocking algorithm to exploit the computation reuse and reduce the memory usage. By computing only $\mathbf{h}^{2}$ and $\mathbf{h}^{3}$ in every step and reusing them in adjacent planes, the amount of computation is reduced approximately by half compared with the $2.5 \mathrm{D}$ basic blocking method. To further reuse the data from the loaded planes in the LDM, a circular array is designed. In this way, among the five planes needed for processing the current plane, four of them can be reused for the next plane. 


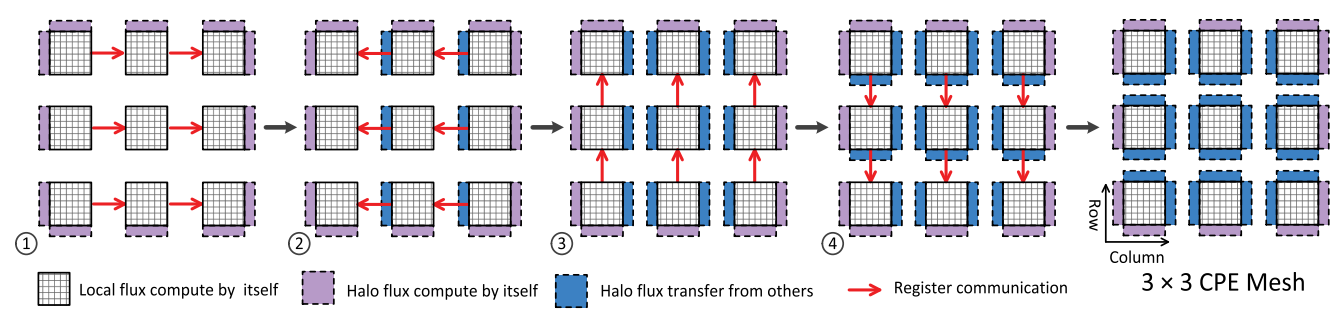

Fig. 5. A conflict-free register communication scheme to exchange halo fluxes between neighboring CPEs. We take a $3 \times 3$ CPE mesh as an example; there are four steps in the arrangement of communications: (1) from left to right, (2) from right to left, (3) from bottom to top, and (4) from top to bottom.

Furthermore, the computation of the current plane can be overlapped with the prefetch of the data for the next plane by using a pipelined double-buffering approach.

\subsection{Conflict-Free Data Exchanging}

For stencil computation, a proper utilization of thread blocking can usually help improve the data locality while exploiting the thread-level parallelism. However, it also gives rise to redundant computations that may substantially degrade the performance. Ideally, the ratio of the redundant computations can be reduced by enlarging the block size. But the size of the local cache, which in this case is the LDM, has very limited capacity. Considering that the main workload for the WENO stencil is the construction of numerical fluxes, we need to seek a way to overlap the intermediate numerical fluxes among adjacent CPEs.

The register communication mechanism supported by SW26010 provides the opportunity to efficiently exchange data between different CPEs. In particular, it supports data exchange among $\mathrm{CPEs}$ that are either in the same row or column in the CPE cluster. To avoid the redundant computation of the numerical fluxes, a conflict-free communication strategy is needed. We should avoid concurrent sending from multiple $\mathrm{CPEs}$ to a same $\mathrm{CPE}$, otherwise an imbalanced usage of the register communication network will occur. In addition, the register communication is a synchronized operation, which means that the CPE that sends the data is blocked until the data are received. Although this restriction can be relaxed by the built-in hardware buffer, too many messages will still congest the data flow.

Based on the above considerations, we propose an efficient and conflict-free data exchange scheme. The details are illustrated in Figure 5, in which a simplified $3 \times 3 \mathrm{CPE}$ cluster is taken as an example. As shown in Figure 5, there are four register communication steps in the data exchange scheme, corresponding to four data-flow paths: (1) from left to right, (2) from right to left, (3) from bottom to top, and (4) from top to bottom. In any step of the conflict-free scheme, each $\mathrm{CPE}$ has at most one send and one receive to communicate with other CPEs. Moreover, each $\mathrm{CPE}$ (except the boundary ones) is designed to send only one vector while receiving one vector at every moment of the transmission, thus avoiding jamming the data flow. After the four steps, every $\mathrm{CPE}$ would have fully exchanged data for the halo fluxes with its neighbors. A good balance and efficiency can be achieved for data exchanging and the method can be generalized to a CPE cluster of any size.

\subsection{Load-Balanced Collaborative Computation}

Based on the conflict-free data exchange, the computation can be done in a collaborative way, in which the redundant computation of numerical fluxes are avoided. Another advantage of this method is that, for the CPEs that are not on the boundary, the halo width can be reduced from 3 


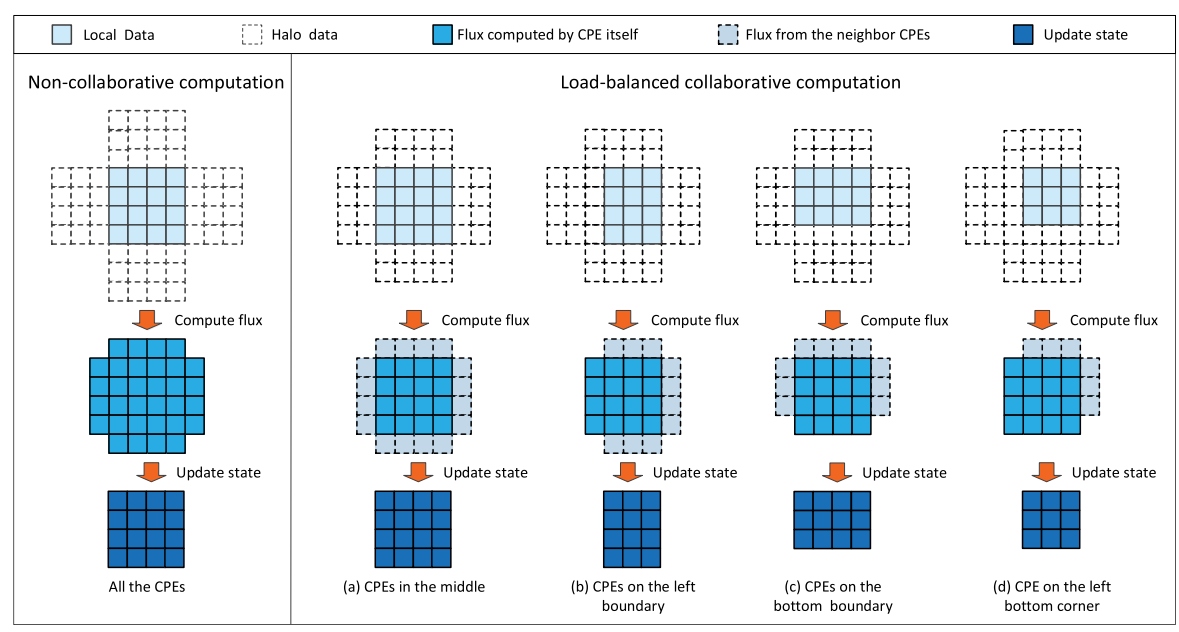

Fig. 6. Illustrations of the data layouts on different CPEs for the non-collaborative computation and loadbalanced collaborative computation.

to 2, therefore the total amount of memory access as well as the usage of the LDM space are also reduced. Despite the above advantages, the collaborative computation designed upon the conflictfree data exchanging scheme also leads to computation imbalance between the inner CPEs and the on-boundary CPEs. For example, as shown in the second row of Figure 5, an on-boundary CPE needs to compute both the local fluxes and the halo fluxes, while an inner CPE only needs to compute the local fluxes. The inner CPEs have to wait for the on-boundary CPEs, and can receive their messages only after the on-boundary CPEs finish their computation. Furthermore, the amount of DMA operations for the inner CPEs and the on-boundary ones are also different because the flux computation on the data chunk boundary requires an extra layer of data.

To alleviate the load imbalance issues mentioned above, we design a load-balanced collaborative computation method, in which the assigned data size is adjusted depending on whether the CPE is on the boundary of the CPE mesh or not. As a result, the number of numerical fluxes computed on each CPE is maintained balanced, and the same amount of data are accessed by each CPE through DMA. Although the workload for updating the final state may be a little different for each CPE, we can ignore the difference since the computation of updating the final state is a relatively small portion of the whole computation.

As a summary, a detailed comparison between the basic non-collaborative computation method and the load-balanced collaborative computation method based on the conflict-free data exchange is illustrated in Figure 6, in which, the case of $4 \times 4$ thread block is taken as an example. In the figure, the left panel corresponds to the non-collaborative computation method, while the right panel corresponds to the different cases for the load-balanced collaborative computation method. On the right panel, it can be seen clearly that the amount of the loaded data and the computation load for numerical fluxes are the same for all the CPEs, indicating that the load balance is well maintained. In addition, both the overall data access and the flux evaluations are reduced compared to the basic non-collaborative case, since the halo fluxes are obtained from neighboring CPEs.

\subsection{Component-Reused Vectorization}

Since the CPE is a reduced 64-bit RISC core with 256-bit vector units, the performance of the optimized code can be further improved by SIMD vectorization. A simple solution of vectorization is 


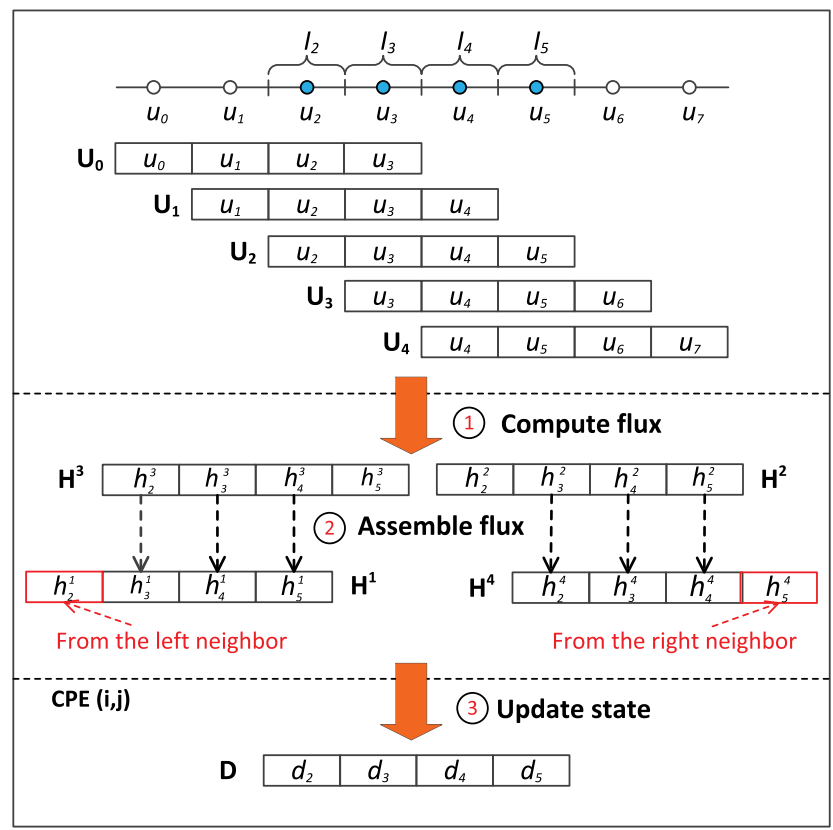

Fig. 7. Illustrations of the SIMD vectorization strategy for the high-order WENO stencil.

just using vector instructions for the major arithmetic operations. However, we found that there are opportunities for data and computation reuse. Therefore, we propose a transformed computation pattern to facilitate more efficient SIMD vectorization.

Figure 7 illustrates the vectorization strategy along the $x$-direction of one row in a block. It is observed that the original dataset is $\left\{\mathbf{u}_{0}, \mathbf{u}_{1}, \ldots, \mathbf{u}_{7}\right\}$, which includes both the local data $\left\{\mathbf{u}_{2}, \mathbf{u}_{3}, \mathbf{u}_{4}, \mathbf{u}_{5}\right\}$ and the halo data $\left\{\mathbf{u}_{0}, \mathbf{u}_{1}\right\}$ and $\left\{\mathbf{u}_{6}, \mathbf{u}_{7}\right\}$. The local flux computation on intervals $I_{2}$ through $I_{5}$ depends on data in $\left\{\mathbf{u}_{0}, \mathbf{u}_{1}, \ldots, \mathbf{u}_{7}\right\}$ in the following pattern:

$$
\begin{aligned}
&\left\{\mathbf{h}_{2}^{2}, \mathbf{h}_{2}^{3}\right\} \leftarrow\left\{\mathbf{u}_{0}, \mathbf{u}_{1}, \mathbf{u}_{2}, \mathbf{u}_{3}, \mathbf{u}_{4}\right\}, \\
&\left\{\mathbf{h}_{3}^{2}, \mathbf{h}_{3}^{3}\right\} \leftarrow\left\{\mathbf{u}_{1}, \mathbf{u}_{2}, \mathbf{u}_{3}, \mathbf{u}_{4}, \mathbf{u}_{5}\right\}, \\
&\left\{\mathbf{h}_{4}^{2}, \mathbf{h}_{4}^{3}\right\} \leftarrow\left\{\mathbf{u}_{2}, \mathbf{u}_{3}, \mathbf{u}_{4}, \mathbf{u}_{5}, \mathbf{u}_{6}\right\}, \\
&\left\{\mathbf{h}_{5}^{2}, \mathbf{h}_{5}^{3}\right\} \leftarrow\left\{\mathbf{u}_{3}, \mathbf{u}_{4}, \mathbf{u}_{5}, \mathbf{u}_{6}, \mathbf{u}_{7}\right\},
\end{aligned}
$$

where the two fluxes on the left rely on the same dataset on the right in each row. Based on this relationship, we can arrange the data into the following five vectors:

$$
\begin{aligned}
\mathbf{U}_{0} & =\left(\mathbf{u}_{0}, \mathbf{u}_{1}, \mathbf{u}_{2}, \mathbf{u}_{3}\right), \\
\mathbf{U}_{1} & =\left(\mathbf{u}_{1}, \mathbf{u}_{2}, \mathbf{u}_{3}, \mathbf{u}_{4}\right), \\
\mathbf{U}_{2} & =\left(\mathbf{u}_{2}, \mathbf{u}_{3}, \mathbf{u}_{4}, \mathbf{u}_{5}\right), \\
\mathbf{U}_{3} & =\left(\mathbf{u}_{3}, \mathbf{u}_{4}, \mathbf{u}_{5}, \mathbf{u}_{6}\right), \\
\mathbf{U}_{4} & =\left(\mathbf{u}_{4}, \mathbf{u}_{5}, \mathbf{u}_{6}, \mathbf{u}_{7}\right),
\end{aligned}
$$

where $\mathrm{U}_{0}$ corresponds to the leftmost components of the four dependent datasets, $\mathrm{U}_{1}$ corresponds to the second leftmost dependent components and so forth. With the five vectors, we can get the 
two flux vectors corresponding to intervals $I_{2}$ through $I_{5}$, denoted as

$$
\begin{aligned}
& \mathbf{H}^{2}=\left(\mathbf{h}_{2}^{2}, \mathbf{h}_{3}^{2}, \mathbf{h}_{4}^{2}, \mathbf{h}_{5}^{2}\right), \\
& \mathbf{H}^{3}=\left(\mathbf{h}_{2}^{3}, \mathbf{h}_{3}^{3}, \mathbf{h}_{4}^{3}, \mathbf{h}_{5}^{3}\right) .
\end{aligned}
$$

During the flux computation, there are plenty of possibilities to reuse their shared components in virtue of the structure of the five input vectors. For example, in Lax-Friedrichs splitting, we only need to do the flux splitting for vector $\mathbf{U}_{0}$ and $\mathbf{U}_{4}$, the flux splitting results for the other three vectors can be assembled from them. In addition, the other two flux vectors $\mathbf{H}^{1}=\left(\mathbf{h}_{2}^{1}, \mathbf{h}_{3}^{1}, \mathbf{h}_{4}^{1}, \mathbf{h}_{5}^{1}\right)$ and $\mathbf{H}^{4}=\left(\mathbf{h}_{2}^{4}, \mathbf{h}_{3}^{4}, \mathbf{h}_{4}^{4}, \mathbf{h}_{5}^{4}\right)$ can be assembled through a series of fast shuffle instructions by reusing the components of the two local flux vectors and the components of those from its neighbors. When these flux vectors are ready, the final state vectors can be constructed directly with them.

\subsection{Other Optimizations}

To further improve the performance, we fuse the three subroutines for Lax-Friedrichs splitting, flux computation, and final state update. Therefore, in the whole WENO computation, only one AoS-to-SoA transformation and one SoA-to-AoS transformation are needed, with the former done at the beginning and the latter at the end [1]. As a result, less vector load and store operations are required, thus the total overhead is reduced. At the inter-process communication level, to reduce the overhead of local memory copy in halo exchange, we implement a customized fast local memory copy of the non-halo data on the CPE cluster with the assistance of DMA. Besides, there are several operations involving vector updates in the explicit Runge-Kutta time integration. Instead of transferring the intermediate vectors back and forth between the main memory and LDM, we fuse the vector update procedure with the WENO stencil computation on the CPE cluster. By doing this, we can reduce the overall memory footprints and at the same time reduce the overhead of thread spawning.

\section{PERFORMANCE MODELING}

In this section, we provide a performance model to analyze the reductions of floating-point operations and memory footprints introduced by our optimizations. To focus on the discussion, we only provide the performance analysis of an interior thread block of size $b_{x} \times b_{y} \times M_{z}$ and halo width $W_{h}$

\subsection{Floating-Point Operations Reductions}

For the three major loops in Algorithm 1, we denote the amount of floating-point operations within each iteration as $F_{1}, F_{2}, F_{3}$, respectively. We then break down the whole thread block into 1D strips along the direction. The number of the strips is denoted as $N_{s}=\left(b_{x} b_{y} M_{z}\right) / \ell$, where $\ell$ is the length of each strip, varying in different situations. According to Algorithm 1, the three loops iterate $\ell+2 W_{h}, \ell+S$ and $\ell$ times, respectively. Here $S$ is a switch controlling whether the halo fluxes are explicitly computed $(S=1$ because there are two single-sided fluxes in each direction and the work amount of each single-sided flux is half of normal) or transferred from a neighboring thread ( $S=0$ because no computation is required). The total amount of floating-point operations in 1D for the thread block can be abstracted as

$$
F=\left[\left(\ell+2 W_{h}\right) F_{1}+(\ell+S) F_{2}+\ell F_{3}\right] N_{s} .
$$

For the $z$-direction, the parameters are set to $\ell=1, W_{h}=3, S=1, N_{s}=b_{x} b_{y} M_{z}$ with the $2.5 \mathrm{D}$ basic blocking and $\ell=M_{z}, W_{h}=3, S=1, N_{s}=b_{x} b_{y}$ with the dependency-reduced blocking method. Therefore, the reduction rate of the floating-point operations from the basic version $F_{z_{-} \text {basic }}$ to the 
optimized one $F_{z \_o p t}$ is

$$
R_{z_{-} \text {flop }}=\frac{F_{z_{-} \text {basic }}-F_{z_{-} o p t}}{F_{z_{-} \text {basic }}}=\frac{\left(6 F_{1}+F_{2}\right)\left(M_{z}-1\right)}{\left(7 F_{1}+2 F_{2}+F_{3}\right) M_{z}} .
$$

For the $x$-direction, the parameters are set to $\ell=b_{x}, W_{h}=3, S=1, N_{s}=b_{y} M_{z}$ with the noncollaborative computation method and $\ell=b_{x}, W_{h}=2, S=0, N_{s}=b_{y} M_{z}$ with the load-balanced collaborative computation. And the reduction rate of the floating-point operations from the former $F_{x_{-} \text {basic }}$ to the latter $F_{x_{-} \text {opt } t}$ is

$$
R_{x_{-} \text {flop }}=\frac{F_{x_{-} \text {basic }}-F_{x_{-} o p t}}{F_{x_{-} \text {basic }}}=\frac{2 F_{1}+F_{2}}{\left(b_{x}+6\right) F_{1}+\left(b_{x}+1\right) F_{2}+b_{x} F_{3}} .
$$

The equation is the same for the $y$-direction except replacing $b_{x}$ with $b_{y}$.

\subsection{Memory Footprints Reductions}

To perform the stencil computation on a thread block, the CPE needs to load both the inner and the halo data into the LDM through DMA. After the computation, the updated inner part will be put back to the main memory via DMA. The amount of loaded memory for a block is

$$
N=\left(b_{x}+2 W_{h}\right)\left(b_{y}+2 W_{h}\right)\left(M_{z}+6\right) N_{\text {bytes }},
$$

where $N_{\text {bytes }}$ denotes the number of bytes for each mesh cell (six double variables), i.e., 48 bytes. Given $b_{x}, b_{y}$ and $M_{z}$, the number $N$ depends on the halo width $W_{h}$. As described in Section 5.1, $W_{h}$ is reduced from 3 to 2 thanks to the load-balanced collaborative computation. We denote the loaded memory footprints of the non-collaborative computation method $\left(W_{h}=3\right)$ as $N_{\text {basic }}$; and that of the load-balanced collaborative computation $\left(W_{h}=2\right)$ as $N_{o p t}$. The reduction rate of loaded memory footprints is therefore

$$
R_{\text {bytes }}=\frac{N_{\text {basic }}-N_{o p t}}{N_{\text {basic }}}=\frac{2\left(b_{x}+b_{y}\right)+20}{b_{x} b_{y}+6\left(b_{x}+b_{y}\right)+36} .
$$

\subsection{Block Size Determination}

In our dependency-reduced blocking method, at least six data planes are required by each CPE, with five for computation and one for prefetching. By further employing the load-balanced collaborative computation, the halo width $W_{h}$ can be reduced to 2 . So the input memory $M_{\text {in }}$ can be expressed as $M_{i n}=6\left(b_{x}+4\right)\left(b_{y}+4\right) N_{\text {bytes }}$. As to the output data, we need four planes, with one for the current plane and the other three for later reuse, so we have $M_{\text {out }}=4 b_{x} b_{y} N_{\text {bytes }}$. Some extra spaces need to be allocated for the intermediate variables shared by the different loops, which occupy $M_{\text {int }}=b_{x} b_{y} N_{i n t}$, where $N_{i n t}$ stands for the number of bytes for these variables. Within the subroutines, about $M_{t m p}=12 \mathrm{~KB}$ space is used to store the temporary vectors. Additionally, we reserve about $M_{\text {others }}=8 \mathrm{~KB}$ memory for the constants, the on-chip fast math library, and other local variables. Since all the above data required by each block should not exceed the LDM capacity (64KB), we can get the constraint

$$
M_{\text {in }}+M_{\text {out }}+M_{\text {int }}+M_{\text {tmp }}+M_{\text {others }}<64 \times 1024 .
$$

Here we also want to ensure $b_{x} \geq b_{y}$ to improve the memory bandwidth utilization, as the $\mathrm{x}$ direction is the most continuous. Based on the above discussion, we can get three possible configurations for $b_{x}$ and $b_{y}$ : (1) $b_{x}=14, b_{y}=1$; (2) $b_{x}=8, b_{y}=2$; (3) $b_{x}=4, b_{y}=4$. To make a better decision, we define the effective region to be the inner part, i.e., $W_{e}=b_{x} b_{y}$, and the effective region ratio to be the inner part divided by the total, i.e., $R_{e f f}=\frac{b_{x} b_{y}}{\left(b_{x}+4\right)\left(b_{y}+4\right)}$. From the comparison shown in Table 1, it can be seen that the third case is the optimal choice among the three considering 
Table 1. Comparisons Among the Three Cases with the Optimal Methods

\begin{tabular}{cccccc}
\hline$b_{x} \times b_{y}$ & $W_{\text {eff }}$ & $R_{\text {eff }}$ & $N_{\text {opt }} /\left(M_{z}+6\right)$ & $F_{x_{-} o p t} / M_{z}$ & $F_{y_{\_} o p t} / M_{z}$ \\
\hline $14 \times 1$ & 14 & $16 \%$ & 4320 bytes & $18 F_{1}+14 F_{2}+14 F_{3}$ & $70 F_{1}+14 F_{2}+14 F_{3}$ \\
\hline $8 \times 2$ & 16 & $22 \%$ & 3456 bytes & $24 F_{1}+16 F_{2}+16 F_{3}$ & $48 F_{1}+16 F_{2}+16 F_{3}$ \\
\hline $4 \times 4$ & 16 & $25 \%$ & 3072 bytes & $32 F_{1}+16 F_{2}+16 F_{3}$ & $32 F_{1}+16 F_{2}+16 F_{3}$ \\
\hline
\end{tabular}

$W_{\text {eff }}$ is the effective region, $R_{\text {eff }}$ is the effective region ratio, $N_{o p t}$ is the amount of loaded memory, and $F_{X_{-} o p t}$ and $F_{y_{-} o p t}$ are the amounts of floating-point operations in the $x$ - and $y$-directions, respectively.

the size of the effective region, and the reductions of memory footprints and floating-point operations. So we choose the third case as the default setting in our study. As a result, with $F_{2} \approx 12 F_{1}$ and $F_{2} \approx 120 F_{3}$, the reduction rate of the floating-point operations is about $20 \%$ in the $x$ - and $y$ directions, and about $58 \%$ in the $z$-direction. And the loaded memory footprints is reduced by $36 \%$.

\section{VALIDATION AND PERFORMANCE EVALUATION}

To accurately measure the performance of our optimized code, we count the number of floatingpoint operations by using the following three methods:

- A manual count of the double-precision arithmetic operations in the code.

- An estimate of the double-precision arithmetic operations based on analysis of the assembly code.

- A direct measurement by running the code with a hardware performance monitor, PERF, which is provided by the vendor of the Sunway TaihuLight supercomputer.

The second and the third methods provide almost identical flop count of the application, while the result of the first method is higher. This is possibly due to compiler optimizations. In our study, we employ the third method (the PERF tool) to measure the floating-point operations.

\subsection{Test Cases}

We validate the correctness and examine the accuracy of high-order WENO simulation code for wave detonations with two test cases, in which the parameters are all set to $\gamma=1.2, q=50, \bar{T}=20$, $\bar{K}=2410.2$.

6.1.1 Example I. In this test case, some proper source terms (functions of $x, y, z$, and $t$ ) are added so that the PDE system Equation (1) has an exact solution, which is

$$
\begin{aligned}
& p(x, y, z, t)=1 \\
& \rho(x, y, z, t)=1+\frac{1}{2} \sin (\pi(x+y+z-3 t)), \\
& u(x, y, z, t)=v(x, y, z, t)=w(x, y, z, t)=1, \\
& Y(x, y, z, t)=\frac{1}{2}+\frac{1}{2} \sin (\pi(x-2 y+2 z-t)) .
\end{aligned}
$$

The computation is done on a $[0,4] \times[0,4] \times[0,4]$ cubic domain with periodic boundary conditions. We use a uniform $N_{x} \times N_{y} \times N_{z}\left(N_{x}=N_{y}=N_{z}=N\right)$ mesh and conduct the simulation till $t=2$. Table 2 shows the difference between the simulated results of the original MPE-only reference code and those of the optimized code, measured in various vector norms. From the table, we can see that the two results are in good agreement, indicating that the optimized code does not exhibit any severe accuracy degradation. In Table 3 we also provide results to examine the order of 
Table 2. Solution Discrepancies of the Reference and Optimized Codes

\begin{tabular}{cccc}
\hline $\mathrm{N}$ & $L^{\infty}$ error & $L^{1}$ error & $L^{2}$ error \\
\hline 30 & $1.64 \times 10^{-13}$ & $1.94 \times 10^{-14}$ & $3.08 \times 10^{-14}$ \\
\hline 60 & $7.51 \times 10^{-13}$ & $2.13 \times 10^{-14}$ & $5.25 \times 10^{-14}$ \\
\hline 120 & $1.27 \times 10^{-12}$ & $2.41 \times 10^{-14}$ & $7.97 \times 10^{-14}$ \\
\hline 240 & $4.98 \times 10^{-13}$ & $1.07 \times 10^{-14}$ & $3.41 \times 10^{-14}$ \\
\hline
\end{tabular}

Table 3. Errors and Orders of Accuracy for the Density Component

\begin{tabular}{ccccccc}
\hline $\mathrm{N}$ & $L^{\infty}$ error & Order & $L^{1}$ error & Order & $L^{2}$ error & Order \\
\hline 30 & $2.40 \times 10^{-2}$ & & $1.72 \times 10^{-2}$ & & $1.85 \times 10^{-2}$ & \\
\hline 60 & $1.07 \times 10^{-3}$ & 4.49 & $7.26 \times 10^{-4}$ & 4.56 & $7.82 \times 10^{-4}$ & 4.56 \\
\hline 120 & $3.83 \times 10^{-5}$ & 4.80 & $2.25 \times 10^{-5}$ & 5.01 & $2.51 \times 10^{-5}$ & 4.96 \\
\hline 240 & $1.28 \times 10^{-6}$ & 4.90 & $7.24 \times 10^{-7}$ & 4.96 & $8.10 \times 10^{-7}$ & 4.96 \\
\hline
\end{tabular}

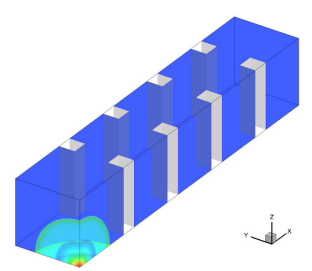

(a) $\mathrm{t}=0.165$

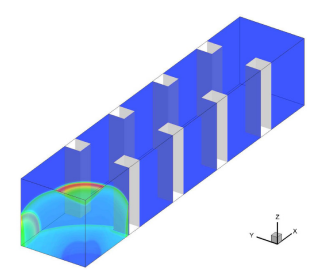

(b) $\mathrm{t}=0.304$

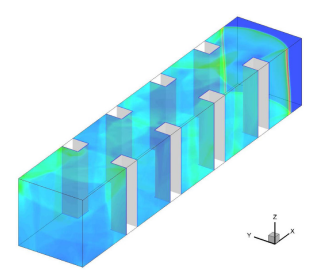

(c) $\mathrm{t}=1.500$

Fig. 8. Pressure distributions of the gaseous wave detonation simulation with multiple obstacles.

accuracy as compared to the analytic solution. To study the accuracy of the spatial discretization, we run the tests on gradually refined meshes. A small fixed time step of size $\Delta t=1 . e-4$ is taken to eliminate the influence from the temporal term. Observations can be made from the table that a fifth-order accuracy is achieved as the mesh is refined.

6.1.2 Example II. As a more realistic test case, example II models gaseous wave detonations in an underground mining tunnel of size $[0,10] \times[0,2] \times[0,2]$. Load-bearing columns, known as obstacles, are located every two length units. Reflective boundary conditions are imposed on all walls except the outlet at $x=10$. The initial condition is

$$
(\rho, \rho E, Y)= \begin{cases}(7,200,0), & \text { if } x^{2}+y^{2}+z^{2} \leq 0.36 \\ (1,55,1), & \text { otherwise }\end{cases}
$$

with zero velocity. We conduct the simulation using a uniform mesh with $\frac{1}{30}$ spatial resolution till $t=1.5$. Some instantaneous results of the pressure are shown in Figure 8 , where we can see that at the early stage of the simulation a precursor shock wave propagates into the unreacted medium with a thin reaction zone immediately behind the shock (Figure 8(a)), then the wave encounters the wall (Figure 8(b)), and finally bypasses the multiple obstacles and approaches the outlet (Figure $8(\mathrm{c})$ ). The simulated results are high resolution, highly accurate, and interpret the underlying physics with great details. With such simulation ability at hand, risk assessments can be made easier for mine engineers and policy makers. 

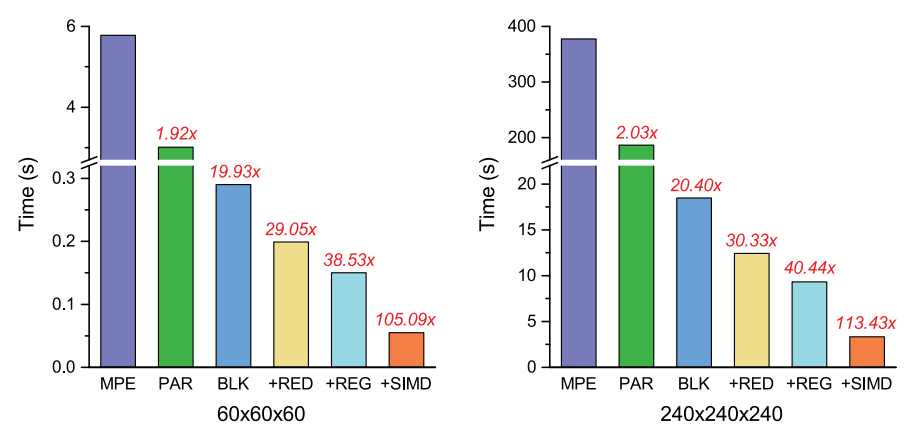

Fig. 9. Measured time cost and the corresponding speedup of the many-core acceleration. Left: $60 \times 60 \times 60$ mesh. Right: $240 \times 240 \times 240$ mesh.

\subsection{Many-Core Acceleration}

Next, we study the performance of the code with various optimization techniques on a single core group of the SW26010 processor. The baseline is chosen as the MPI-based single-threaded reference code that runs on a single MPE. We then measure the performance improvements of five optimized versions, including the multi-threaded version based on a naive memory accessing strategy (PAR), the one with the 2.5D basic blocking (BLK), the one with the dependency-reduced blocking $(+\mathrm{RED})$, the one with further load-balanced collaborative computation based on the register communication $(+\mathrm{REG})$, and the one with the component-reused vectorization (+VEC).

The detailed performance results are shown in Figure 9, in which the left chart corresponds to the results on a $60 \times 60 \times 60$ mesh and the right chart $240 \times 240 \times 240$. Taking the latter as an example, a straightforward parallelization based on the naive memory accessing strategy can only lead to a speedup of $2.03 \mathrm{x}$, which is much smaller than the total number of CPEs. The speedup increases to $20.40 \mathrm{x}$ when the $2.5 \mathrm{D}$ basic blocking is applied, and further to $30.33 \mathrm{x}$ when employing the dependency-reduced blocking strategy. On top of that, a 40.40x speedup is achieved if the load-balanced collaborative computation scheme is adopted based on the conflict-free thread-level data exchanging. The component-reused vectorization further increases the speedup to $113.43 \mathrm{x}$, indicating that vectorization method we use is effective. For the case of the $60 \times 60 \times 60$ mesh, the speedup slightly drops due to the smaller mesh size, but still reaches $105.59 \mathrm{x}$. The sustained performance on a single core group of SW26010 is 178.85 Gflops, which is around $23.38 \%$ of the theoretical peak.

\subsection{Parallel Scalability}

In this subsection, the parallel performance of the optimized simulation code for wave detonation is investigated, including both strong and weak scaling tests.

6.3.1 Strong-Scaling Results. In the strong-scaling tests, we fix the problem size and increase the total number of MPI processes. Two meshes are tested here, including a relatively small one of $3840 \times 1920 \times 480$ mesh cells, denoted as mesh \#1, and a relatively large one of $7680 \times 3840 \times 480$ mesh cells, denoted as mesh \#2. We increase the number of MPI processes from 4,096 to 131,072 by a factor of 2. Testing results in terms of the speedup and the parallel efficiency are shown in Figure 10. For mesh \#2 the parallel efficiency is $62.14 \%$ when 131,072 processes are used, which corresponds to $8,519,680$ cores. For mesh \#1, the parallel efficiency can stay above $60 \%$ when 65,536 processes are utilized, which corresponds to $4,259,840$ cores, but drops to $51.74 \%$ when 131,072 processes are used. This is expected because, as more processes are used, the subdomain size decreases and 


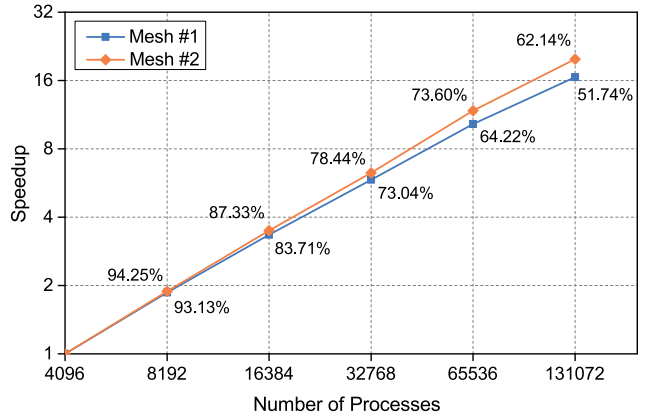

Fig. 10. Strong scaling results of the wave detonation simulation on Sunway TaihuLight, using 4,096 processes to 131,072 processes $(266,240$ cores to $8,519,680$ cores).

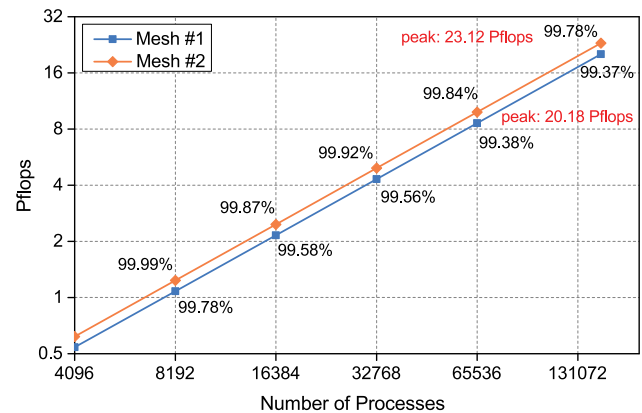

Fig. 11. Weak-scaling results of the wave detonation simulation on Sunway TaihuLight, using 4,096 processes to 153,600 processes $(266,240$ cores to $9,984,000$ cores).

Table 4. Summary of Existing Works Related to Stencil Computations on Sunway TaihuLight-Numbers are Obtained from the Published Papers; Unreported Values are Labelled as “_”

\begin{tabular}{llllll}
\hline Application & Method & Precision & Stencil & Cores & Performance \\
\hline Phase field simulation & explicit & double & second-order 7-point & $10.6 \mathrm{M}$ & 50.58 Pflops \\
\hline Global wave modeling & explicit & double & - & $10.6 \mathrm{M}$ & 45.43 Pflops \\
\hline Atmospheric modeling & explicit & double & second-order 13-point & $10.6 \mathrm{M}$ & 25.96 Pflops \\
& fully implicit & & & & 7.95 Pflops \\
\hline Earthquake simulation & explicit & single & fourth-order 13-point & $10.4 \mathrm{M}$ & 18.9 Pflops \\
\hline CAM model & explicit & double & - & $10.0 \mathrm{M}$ & 3.3 Pflops \\
\hline Our work & explicit & double & fifth-order 19-point & 9.98M & 23.12 Pflops \\
\hline
\end{tabular}

the ratio of communication to computation becomes larger and eventually hinders the overall performance.

6.3.2 Weak-Scaling Results. In the weak-scaling tests, we fix the size of the subdomain and increase the problem size with respect to the total number of MPI processes. We also consider two problem sizes. The larger one corresponds to the subdomain of size $240 \times 240 \times 240$, which is the maximum size to fit into the main memory of a single core group. The smaller one uses a subdomain of size $120 \times 120 \times 120$, which is $1 / 8$ of the larger one. As the number of processes ranges from 4,096 to 153,600 , the total number of unknowns increases from 42.5 billion to 1.59 trillion for the smaller mesh (mesh \#1) and from 339.7 billion to 12.74 trillion for the larger one (mesh \#2). The test results are given in Figure 11. It can be observed that a nearly ideal parallel efficiency is achieved for both cases, even when the number of processes is 153,600, which corresponds to 9.984 million cores. The maximal sustained performance is 20.18 Pflops for mesh \#1 and 23.12 Pflops for mesh \#2, both in double precision. In particular, the flops efficiency with respect to the theoretical peak performance at the ten-million-core scale is as high as $19.68 \%$.

6.3.3 Comparison with Previous Works. For comparison purpose, Table 4 summarizes some existing works related to 3D stencil computations on Sunway TaihuLight, with the numbers collected from literatures. In the phase field simulation [55], a very high performance of over 50 Pflops is achieved, despite the simple second-order 7-point stencil required during the simulation. This is because an exponential integrator method is used for time integration, which relies heavily on dense 
matrix multiplications. The global wave simulation [31] also involves stencil like computations, but the stencil is in an integral form of six overlaid loops, which greatly increases the computation intensity and leads to an aggregate performance of over 45 Pflops. Both explicit and fully implicit time integration approaches are studied in the atmospheric simulation [54] (ACM Gordon Bell Prize in 2016), in which the explicit method is based on a second-order 13-point stencil computation and the implicit one consists of not only stencil but also complex preconditioners and iterative solvers. The earthquake simulation [14] (ACM Gordon Bell Prize in 2017) is based on an explicit method that involves a fourth-order 13-point stencil computation. The reported performance of 18.9 Pflops is high, considering the fact that the high-order stencil is much more complicated than low-order ones, but we remark here that single precision is used instead of the full double precision, which greatly reduces the memory footprints and the occupation of local cache. The work on refactoring the CAM climate model [15] also requires various types of stencil computations, mostly low orders, spreading in various computation subroutines. Our work presented in this article differs from the above works in the way that we conduct an in-depth study on optimizing a real-world high-oder WENO stencil and report double-precision performance that is comparable to the performance of low-order stencils or high-order stencils with reduced arithmetics.

\section{RELATED WORK}

Stencil computation is an important primitive frequently found in many scientific and engineering computing applications. A large variety of optimization techniques for stencil computing have been studied on modern hardware architectures such as CPU [7, 8, 27, 33, 35], GPU [7, 24, 27], Intel Xeon Phi [41, 52, 53], and IBM Cell Processor [7, 8]. For example, methods such as data blocking are popular approaches in the study of the stencil computations, and have been proved a key to successful data reuse [33]. Both cache-aware algorithms and cache-oblivious techniques were well studied to improve the data locality for cache-based architectures [7]. A 3.5D blocking algorithm, which consists of a 2.5D spatial blocking and an additional temporal blocking to increase the opportunities of data reuse, was proposed in Reference [27] on GPUs. A multi-level domain decomposition scheme with a 2.5D blocking was utilized to fully exploit the MPI-level and threadlevel parallelism on Sunway TaihuLight [14]. To exploit the computation reuse, a code generation and performance tuning framework was investigated for the stencil computation on GPUs [25]. For high-order stencil optimizations, a multi-level parallelization framework including inter-node parallelism by spatial decomposition, intra-chip parallelism through multi-threading, and vectorization was applied to a sixth-order stencil on multi-core architectures [11]. To reduce the cost of floating-point operations, studies on compiler-directed transformation by reusing the partial sums for multiple results were carried on constant-coefficient, out-of-place stencils [2]. SIMD parallelization including translocated statement fusion, vector composition via shuffle on multi-core clusters were studied in Reference [28]. All the above works can help us in the implementation and optimization of the high-order WENO stencil on Sunway TaihuLight. In particular, we employ a $2.5 \mathrm{D}$ dependency-reduced blocking methodology with reduced floating-point operations and a component-reused vectorization strategy on variable-coefficient finite different WENO stencil to achieve high performance.

A number of applications have been implemented and optimized on the Sunway TaihuLight supercomputer, such as earthquake simulation [14], atmospheric modeling [1, 16, 54], global surface wave numerical simulation [31], phase field simulation [55], and so on. Among them, various optimization techniques have been adopted to improve the performance. For example, the multithreading technique was employed by most of the applications [1, 9, 12, 16, 21, 22, 31, 54, 55] due to the powerful computing capability of the CPE cluster. The fast DMA channel was utilized in $[9,12,21,22,55]$, and was proved to be more efficient than the naive data accessing method [1]. 
Collaborations between the CPEs were employed in a scalable BFS algorithm [22], in which the $\mathrm{CPEs}$ were partitioned into different groups: producer, consumer, and router, and a contention-free data shuffling scheme was carefully designed based on the fast on-chip register communication. Vectorization was applied to increase the instruction-level parallelism [1, 9, 12, 16, 54]. Pipeline instruction reordering was proposed in $[12,21]$ to explore higher efficiency for the core computing process. Load balance was considered in Reference [31], and was solved by utilizing an irregular quasi-rectangular domain decomposition method. A master-worker cooperative computing workflow was presented in $[1,31]$, which could overlap the computation and communication efficiently. Some other optimizations such as double buffering [12, 21], global memory access reduction [1, 16], efficient use of local memory [16], and nonlinear mathematical functions [16, 31] were also studied to improve the performance. We consider most of the optimization techniques in our study, including multi-threading, DMA utilization, collaborations among CPEs, SIMD, load balancing, double buffering, and so on. In particular, the load-balanced collaborative computing method we propose in this article successfully reduces the halo width from 3 to 2 , which not only greatly improves the effective region ratio within a limited LDM space, but also reduces the floating-point operations, which is an inherent issue for high-order wide stencils on many-core systems with highly limited memory space.

\section{CONCLUDING REMARKS}

It is a challenging task to implement high-order stencils on modern supercomputers, especially on heterogeneous many-core based systems, due to the complexities of the architectures as well as the sophisticated computing and memory behaviors in the high-order stencils. As typical representatives of high-order stencils, WENO methods have been broadly applied in many applications, ranging from computational fluid dynamics [20, 29, 45], astrophysics [13, 32], semiconductor device simulations $[4,5]$, and so on. In this article, we take WENO as an example and propose several optimization techniques to tackle the difficulties between the high-order stencil in 3D wave detonations and the complicated architectures of the Sunway TaihuLight supercomputer. Various optimization techniques are presented, including a dependency-reduced blocking method for improved memory efficiency and computation reuse, a load-balanced collaborative computing approach with conflict-free data exchange for further computation reuse and memory access reduction, and a component-reused vectorization scheme for increased instruction-level parallelism. Experiments show that the optimized WENO stencil computation code can scale in nearly an ideal way to 9.98 million cores, sustaining 23.12 Pflops in double precision and solving problems with 12.74 trillion unknowns. The basic idea behind our efforts can be extended to other high-order stencils, such as the discontinuous Galerkin, among others, and even to some complex non-stencil applications. In addition to that, we believe that some of the key optimization techniques can provide hints on optimizing high-order stencil computations on other heterogeneous many-core based systems.

\section{REFERENCES}

[1] Yulong Ao, Chao Yang, Xinliang Wang, Wei Xue, Haohuan Fu, Fangfang Liu, Lin Gan, Ping Xu, and Wenjing Ma. 2017. 26 PFLOPS stencil computations for atmospheric modeling on sunway taihulight. In Proceedings of the 2017 IEEE International Parallel and Distributed Processing Symposium (IPDPS'17). IEEE, 535-544. DOI : http://dx.doi.org/10. 1109/IPDPS.2017.9

[2] Protonu Basu, Mary Hall, Samuel Williams, Brian Van Straalen, Leonid Oliker, and Phillip Colella. 2015. Compilerdirected transformation for higher-order stencils. In Proceedings of the 2015 IEEE International Parallel and Distributed Processing Symposium (IPDPS’15). IEEE, 313-323. DOI : http://dx.doi.org/10.1109/IPDPS.2015.103

[3] Vitaly Bychkov, Arkady Petchenko, V'yacheslav Akkerman, and Lars-Erik Eriksson. 2005. Theory and modeling of accelerating flames in tubes. Phys. Rev. E 72, 4 (2005), 046307. DOI : http://dx.doi.org/10.1103/PhysRevE.72.046307 
[4] José A. Carrillo, Irene M. Gamba, Armando Majorana, and Chi-Wang Shu. 2003. A WENO-solver for the transients of boltzmann-poisson system for semiconductor devices: Performance and comparisons with Monte Carlo methods. f. Comput. Phys. 184, 2 (2003), 498-525. DOI : http://dx.doi.org/10.1016/S0021-9991(02)00032-3

[5] Carlo Cercignani, Irene M. Gamba, Joseph W. Jerome, and Chi-Wang Shu. 2000. Device benchmark comparisons via kinetic, hydrodynamic, and high-field models. Comput. Meth. Appl. Mech. Eng. 181, 4 (2000), 381-392. DOI : http: //dx.doi.org/10.1016/S0045-7825(99)00186-3

[6] Wang Cheng, Bi Yong, Han Wenhu, and Ning Jianguo. 2013. Large-scale parallel computing for 3D gaseous detonation. In Proceedings of the International Conference on Parallel Computing in Fluid Dynamics. Springer, 541-552. DOI : http://dx.doi.org/10.1007/978-3-642-53962-6_49

[7] Kaushik Datta, Shoaib Kamil, Samuel Williams, Leonid Oliker, John Shalf, and Katherine Yelick. 2009. Optimization and performance modeling of stencil computations on modern microprocessors. SIAM Rev. 51, 1 (2009), 129-159. DOI : http://dx.doi.org/10.1137/070693199

[8] Kaushik Datta, Mark Murphy, Vasily Volkov, Samuel Williams, Jonathan Carter, Leonid Oliker, David Patterson, John Shalf, and Katherine Yelick. 2008. Stencil computation optimization and auto-tuning on state-of-the-art multicore architectures. In Proceedings of the 2008 ACM/IEEE Conference on Supercomputing. IEEE Press, Article 4, 12 pages. DOI : http://dx.doi.org/10.1109/SC.2008.5222004

[9] Wenqian Dong, Letian Kang, Zhe Quan, Kenli Li, Keqin Li, Ziyu Hao, and Xiang-Hui Xie. 2016. Implementing molecular dynamics simulation on sunway taihulight system. In Proceedings of the 2016 IEEE 18th International Conference on High Performance Computing and Communications; IEEE 14th International Conference on Smart City; IEEE 2nd International Conference on Data Science and Systems (HPCC/SmartCity/DSS'16). IEEE, 443-450. DOI : http: //dx.doi.org/10.1109/HPCC-SmartCity-DSS.2016.0070

[10] Hua-Shu Dou, Her Mann Tsai, Boo Cheong Khoo, and Jianxian Qiu. 2008. Simulations of detonation wave propagation in rectangular ducts using a three-dimensional WENO scheme. Combust. Flame 154, 4 (2008), 644-659. DOI : http: //dx.doi.org/10.1016/j.combustflame.2008.06.013

[11] Hikmet Dursun, Ken-ichi Nomura, Liu Peng, Richard Seymour, Weiqiang Wang, Rajiv K. Kalia, Aiichiro Nakano, and Priya Vashishta. 2009. A multilevel parallelization framework for high-order stencil computations. In Proceedings of the European Conference on Parallel Processing. Springer, 642-653. DOI : http://dx.doi.org/10.1007/978-3-642-03869-3_ 61

[12] Jiarui Fang, Haohuan Fu, Wenlai Zhao, Bingwei Chen, Weijie Zheng, and Guangwen Yang. 2017. swDNN: A library for accelerating deep learning applications on sunway taihulight. In Proceedings of the 2017 IEEE International Parallel and Distributed Processing Symposium (IPDPS'17). IEEE, 615-624. DOI : http://dx.doi.org/10.1109/IPDPS.2017.20

[13] Long-Long Feng, Chi-Wang Shu, and Mengping Zhang. 2004. A hybrid cosmological hydrodynamic/N-body code based on a weighted essentially nonoscillatory scheme. Astrophys. F. 612, 1 (2004), 1. DOI : http://dx.doi.org/10.1086/ 422513

[14] Haohuan Fu, Conghui He, Bingwei Chen, Zekun Yin, Zhenguo Zhang, Wenqiang Zhang, Tingjian Zhang, Wei Xue, Weiguo Liu, Wanwang Yin, Guangwen Yang, and Chen Xiaofei. 2017. 18.9-Pflops nonlinear earthquake simulation on sunway taihulight: Enabling depiction of $18-\mathrm{Hz}$ and 8-meter scenarios. In Proceedings of the International Conference for High Performance Computing, Networking, Storage and Analysis. ACM, Article 2, 12 pages. DOI : http://dx.doi.org/ $10.1145 / 3126908.3126910$

[15] Haohuan Fu, Junfeng Liao, Nan Ding, Xiaohui Duan, Lin Gan, Yishuang Liang, Xinliang Wang, Jinzhe Yang, Yan Zheng, Weiguo Liu, Lanning Wang, and Guangwen Yang. 2017. Redesigning CAM-SE for peta-scale climate modeling performance and ultra-high resolution on sunway taihulight. In Proceedings of the International Conference for High Performance Computing, Networking, Storage and Analysis. ACM, Article 1, 12 pages. DOI : http://dx.doi.org/10.1145/ 3126908.3126909

[16] Haohuan Fu, Junfeng Liao, Jinzhe Yang, Lanning Wang, Zhenya Song, Xiaomeng Huang, Chao Yang, Wei Xue, Fangfang Liu, Fangli Qiao, Wei Zhao, Hou Chaofeng Zhang Chenglong Yin, Xunqiang, Wei Ge, Jian Zhang, Yanggang Wang, and Chunbo Zhou. 2016. The sunway taihulight supercomputer: System and applications. Sci. China Inform. Sci. 59, 7 (2016), 072001. DOI : http://dx.doi.org/10.1007/s11432-016-5588-7

[17] S. K. Godunov. 1959. A finite difference method for the computation of discontinuous solutions of the equations of fluid dynamics. Sbornik: Math. 47, 8-9 (1959), 357-393.

[18] Sigal Gottlieb, Chi-Wang Shu, and Eitan Tadmor. 2001. Strong stability-preserving high-order time discretization methods. SIAM Rev. 43, 1 (2001), 89-112. DOI : http://dx.doi.org/10.1137/S003614450036757X

[19] Guang-Shan Jiang and Chi-Wang Shu. 1996. Efficient implementation of weighted ENO schemes. F. Comput. Phys. 126, 1 (1996), 202-228. DOI : http://dx.doi.org/10.1006/jcph.1996.0130

[20] Guang-Shan Jiang and Cheng-chin Wu. 1999. A high-order WENO finite difference scheme for the equations of ideal magnetohydrodynamics. F. Comput. Phys. 150, 2 (1999), 561-594. DOI : http://dx.doi.org/10.2322/tjsass.47.268 
[21] Lijuan Jiang, Chao Yang, Yulong Ao, Wanwang Yin, Wenjing Ma, Qiao Sun, Fangfang Liu, Rongfen Lin, and Peng Zhang. 2017. Towards highly efficient DGEMM on the emerging SW26010 many-core processor. In Proceedings of the 2017 46th International Conference on Parallel Processing (ICPP'17). IEEE, 422-431. DOI : http://dx.doi.org/10.1109/ ICPP.2017.51

[22] Heng Lin, Xiongchao Tang, Bowen Yu, Youwei Zhuo, Wenguang Chen, Jidong Zhai, Wanwang Yin, and Weimin Zheng. 2017. Scalable graph traversal on sunway taihulight with ten million cores. IEEE, 635-645. DOI : http://dx.doi. org/10.1109/IPDPS.2017.53

[23] Xu-Dong Liu, Stanley Osher, and Tony Chan. 1994. Weighted essentially non-oscillatory schemes. F. Comput. Phys. 115, 1 (1994), 200-212. DOI : http://dx.doi.org/10.1006/jcph.1994.1187

[24] Thibaut Lutz, Christian Fensch, and Murray Cole. 2013. PARTANS: An autotuning framework for stencil computation on multi-GPU systems. ACM Trans. Arch. Code Optim. 9, 4, Article 59 (2013), 24 pages. DOI : http://dx.doi.org/10.1145/ 2400682.2400718

[25] Wen-Jing Ma, Kan Gao, and Guo-Ping Long. 2016. Highly optimized code generation for stencil codes with computation reuse for GPUs. F. Comput. Sci. Technol. 31, 6 (2016), 1262-1274. DOI : http://dx.doi.org/10.1007/s11390-016-1696-5

[26] Hans Meuer, Erich Strohmaier, Jack Dongarra, Horst Simon, and Meuer Martin. 2017. Top 500 Supercomputer Lists. Retrieved November 14, 2017 from http://www.top500.org.

[27] Anthony Nguyen, Nadathur Satish, Jatin Chhugani, Changkyu Kim, and Pradeep Dubey. 2010. 3.5-D blocking optimization for stencil computations on modern CPUs and GPUs. In Proceedings of the 2010 International Conference for High Performance Computing, Networking, Storage and Analysis (SC'10). IEEE, 1-13. DOI: http://dx.doi.org/10.1109/ SC. 2010.2

[28] Liu Peng, Richard Seymour, Ken-ichi Nomura, Rajiv K. Kalia, Aiichiro Nakano, Priya Vashishta, Alexander Loddoch, Michael Netzband, William R. Volz, and Chap C. Wong. 2009. High-order stencil computations on multicore clusters. In Proceedings of the IEEE International Symposium on Parallel \& Distributed Processing, 2009 (IPDPS'09). IEEE, 1-11. DOI : http://dx.doi.org/10.1109/IPDPS.2009.5161011

[29] Sergio Pirozzoli. 2004. Dynamics of ring vortices impinging on planar shock waves. Phys. Fluids 16, 5 (2004), 11711185. DOI : http://dx.doi.org/10.1063/1.1649336

[30] Williama Putnam. 2011. Graphics processing unit (GPU) acceleration of the goddard earth observing system atmospheric model. NASA Technical Report, Goddard Space Flight Center. http://ntrs.nasa.gov/search.jsp?R=20120009084.

[31] Fangli Qiao, Wei Zhao, Xunqiang Yin, Xiaomeng Huang, Xin Liu, Qi Shu, Guansuo Wang, Zhenya Song, Xinfang Li, Haixing Liu, Guangwen Yang, and Yeli Yuan. 2016. A highly effective global surface wave numerical simulation with ultra-high resolution. In Proceedings of the SC16: International Conference for High Performance Computing, Networking, Storage and Analysis. IEEE, Article 5, 11 pages. DOI : http://dx.doi.org/10.1109/SC.2016.4

[32] Jing-Mei Qiu, Chi-Wang Shu, Long-Long Feng, and Li-Zhi Fang. 2006. A WENO algorithm for the radiative transfer and ionized sphere at reionization. New Astron. 12, 1 (2006), 1-10. DOI : http://dx.doi.org/10.1016/j.newast.2006.04.007

[33] Gabriel Rivera and Chau-Wen Tseng. 2000. Tiling optimizations for 3D scientific computations. In Proceedings of the Supercomputing, ACM/IEEE 2000 Conference. IEEE Computer Society, Article 32. DOI : http://dx.doi.org/10.1109/SC. 2000.10015

[34] Philip L. Roe. 1981. Approximate Riemann solvers, parameter vectors, and difference schemes. f. Comput. Phys. 43, 2 (1981), 357-372. DOI : http://dx.doi.org/10.1016/0021-9991(81)90128-5

[35] Shimpei Sato, Yukinori Sato, and Toshio Endo. 2015. Investigating potential performance benefits of memory layout optimization based on roofline model. In Proceedings of the 2nd International Workshop on Software Engineering for Parallel Systems. ACM, 50-56. DOI : http://dx.doi.org/10.1145/2837476.2837483

[36] J. Shepherd, F. Pintgen, J. Austin, and C. Eckett. 2002. The structure of the detonation front in gases. In 40th AIAA Aerospace Sciences Meeting \& Exhibit. 773. DOI : http://dx.doi.org/10.2514/6.2002-773

[37] Takashi Shimokawabe, Tomohiro Takaki, Toshio Endo, Akinori Yamanaka, Naoya Maruyama, Takayuki Aoki, Akira Nukada, and Satoshi Matsuoka. 2011. Peta-scale phase-field simulation for dendritic solidification on the TSUBAME 2.0 supercomputer. In 2011 International Conference for High Performance Computing, Networking, Storage and Analysis (SC'11). IEEE, 1-11. DOI : http://dx.doi.org/10.1145/2063384.2063388

[38] Chi-Wang Shu. 2009. High order weighted essentially nonoscillatory schemes for convection dominated problems. SIAM Rev. 51, 1 (2009), 82-126. DOI : http://dx.doi.org/10.1137/070679065

[39] Mohammed Sourouri, Scott B. Baden, and Xing Cai. 2017. Panda: A compiler framework for concurrent CPU+ GPU execution of 3D stencil computations on GPU-accelerated supercomputers. Int. F. Parallel Program. 45, 3 (2017), 711729. DOI : http://dx.doi.org/10.1007/s10766-016-0454-1

[40] R. A. Strehlow. 1970. Multi-dimensional detonation wave structure. Astronautica Acta 15 (1970), 345-357.

[41] Lukasz Szustak, Krzysztof Rojek, and Pawel Gepner. 2013. Using Intel Xeon Phi coprocessor to accelerate computations in MPDATA algorithm. In Proceedings of the International Conference on Parallel Processing and Applied Mathematics. Springer, 582-592. DOI : http://dx.doi.org/10.1007/978-3-642-55224-3_54 
[42] Lukasz Szustak, Krzysztof Rojek, Tomasz Olas, Lukasz Kuczynski, Kamil Halbiniak, and Pawel Gepner. 2015. Adaptation of MPDATA heterogeneous stencil computation to Intel Xeon Phi coprocessor. Sci. Program. 2015 (2015), 10. DOI : http://dx.doi.org/10.1155/2015/642705

[43] Lukasz Szustak, Krzysztof Rojek, Roman Wyrzykowski, and Pawel Gepner. 2014. Toward efficient distribution of MPDATA stencil computation on intel MIC architecture. Proceedings of HiStencils 14 (2014), 51-56.

[44] Sirui Tan, Cheng Wang, Chi-Wang Shu, and Jianguo Ning. 2012. Efficient implementation of high order inverse laxwendroff boundary treatment for conservation laws. f. Comput. Phys. 231, 6 (2012), 2510-2527. D0I : http://dx.doi.org/ 10.1016/j.jcp.2011.11.037

[45] Susumu Teramoto. 2005. Large eddy simulation of shock wave/boundary layer interaction. Trans. Jpn. Soc. Aeronaut. Space Sci. 47, 158 (2005), 268-275.

[46] Cheng Wang, XinZhuang Dong, and Chi-Wang Shu. 2015. Parallel adaptive mesh refinement method based on WENO finite difference scheme for the simulation of multi-dimensional detonation. f. Comput. Phys. 298 (2015), 161-175. DOI : http://dx.doi.org/10.1016/j.jcp.2015.06.001

[47] Cheng Wang, Tianbao Ma, and Jie Lu. 2010. Influence of obstacle disturbance in a duct on explosion characteristics of coal gas. SCIENCE CHINA Phys. Mech. Astron. 53, 2 (2010), 269-278. DOI : http://dx.doi.org/10.1007/s11433-009-0270-3

[48] Cheng Wang, Chi-Wang Shu, Wenhu Han, and Jianguo Ning. 2013. High resolution WENO simulation of 3D detonation waves. Combustion and Flame 160, 2 (2013), 447-462. DOI : http://dx.doi.org/10.1016/j.combustflame.2012.10.002

[49] Cheng Wang, Xiangxiong Zhang, Chi-Wang Shu, and Jianguo Ning. 2012. Robust high order discontinuous galerkin schemes for two-dimensional gaseous detonations. f. Comput. Phys. 231, 2 (2012), 653-665. DOI : http://dx.doi.org/10. 1016/j.jcp.2011.10.002

[50] Fabian Wermelinger, Babak Hejazialhosseini, Panagiotis Hadjidoukas, Diego Rossinelli, and Petros Koumoutsakos. 2016. An efficient compressible multicomponent flow solver for heterogeneous CPU/GPU architectures. In Proceedings of the Platform for Advanced Scientific Computing Conference. ACM, 8. DOI : http://dx.doi.org/10.1145/2929908. 2929914

[51] Zhao Xiangdi, Jiang Chunming, Wang Yanping, Yuan Jiwu, and Wang Zheng. 2014. Simulation study on an explosion accident in china. Process Saf. Prog. 33, 1 (2014), 56-63. DOI : http://dx.doi.org/10.1002/prs.11620

[52] Wei Xue, Chao Yang, Haohuan Fu, Xinliang Wang, Yangtong Xu, Lin Gan, Yutong Lu, and Xiaoqian Zhu. 2014. Enabling and scaling a global shallow-water atmospheric model on tianhe-2. In Proceedings of the 2014 IEEE 28th International Parallel and Distributed Processing Symposium. IEEE, 745-754. DOI : http://dx.doi.org/10.1109/IPDPS.2014.82

[53] Wei Xue, Chao Yang, Haohuan Fu, Xinliang Wang, Yangtong Xu, Junfeng Liao, Lin Gan, Yutong Lu, Rajiv Ranjan, and Lizhe Wang. 2015. Ultra-scalable CPU-MIC acceleration of mesoscale atmospheric modeling on Tianhe-2. IEEE Trans. Comput. 64, 8 (2015), 2382-2393. DOI : http://dx.doi.org/10.1109/TC.2014.2366754

[54] Chao Yang, Wei Xue, Haohuan Fu, Hongtao You, Xinliang Wang, Yulong Ao, Fangfang Liu, Lin Gan, Ping Xu, Lanning Wang, Guangwen Yang, and Weimin Zheng. 2016. 10M-core scalable fully-implicit solver for nonhydrostatic atmospheric dynamics. In Proceedings of the International Conference for High Performance Computing, Networking, Storage and Analysis (SC'16). IEEE, Article 6, 12 pages. DOI : http://dx.doi.org/10.1109/SC.2016.5

[55] Jian Zhang, Chunbao Zhou, Yangang Wang, Lili Ju, Qiang Du, Xuebin Chi, Dongsheng Xu, Dexun Chen, Yong Liu, and Zhao Liu. 2016. Extreme-scale phase field simulations of coarsening dynamics on the sunway taihulight supercomputer. In Proceedings of the International Conference for High Performance Computing, Networking, Storage and Analysis. IEEE Press, Article 4, 12 pages. DOI : http://dx.doi.org/10.1109/SC.2016.3

[56] Xiangxiong Zhang and Chi-Wang Shu. 2012. Positivity-preserving high order finite difference WENO schemes for compressible Euler equations. F. Comput. Phys. 231, 5 (2012), 2245-2258. DOI : http://dx.doi.org/10.1016/j.jcp.2011.11. 020

Received February 2018; accepted April 2018 